\title{
Vibratory monitoring of gear transmissions in variable regime
}

\author{
Mustapha Merzoug ${ }^{1, a}$, Khalid Ait SghiR ${ }^{2}$, Abdelhamid Miloudi ${ }^{1}$ And \\ JeAn PAUl Dron ${ }^{2}$ \\ 1 Laboratoire de Mécanique Avancée, USTHB, BP 32 El Alia, 16111 Bab Ezzouar, Alger, Algerie \\ 2 Groupe de Recherche en Science Pour l'Ingénieur, Université de Reims Champagne Ardenne, UFR Sciences Exactes \\ et Naturelles, Moulin de la Housse, BP 1039, 51687 Reims Cedex, France
}

Received 9 January 2014, Accepted 15 May 2014

\begin{abstract}
This paper falls within the context of diagnosis of rotating machines in speed variable regime. Based on simulation signals, this work has the purpose to find relevant indicators for the diagnosis of gear transmissions in a variable regime. Two indicators are proposed; the first indicator is the RMS value applied to the vibration signal divided by its corresponding instantaneous frequency. The second is the normalised gear frequency by averaging speed. The gear frequency and averaging speed are estimated from the spectrogram. To test the proposed indicators simulate signals have been used. These signals are the results of a dynamic modelling of the gears transmission and are calculated by using the Newmark integration diagram. This dynamic modelling takes into account the eccentricity defect.
\end{abstract}

Key words: Gears / instantaneous frequency / RMS value / spectral analysis

\section{Nomenclature}

\begin{tabular}{|ll|}
\hline$e_{p}$ and $e_{g}$ & Wheel eccentricities \\
$R_{p}$ and $R_{g}$ & Radii of the wheels base circles \\
$\theta_{p}$ and $\theta_{g}$ & Rotation angles of wheels \\
$y_{p}$ and $y_{g}$ & Displacements of shaft supports \\
$\Omega_{1}$ & Drive shaft frequency \\
$\Omega_{2}$ & Driven shaft frequency \\
$M_{p}$ and $M_{g}$ & Masses of the pinion and gear \\
$I_{p}$ and $I_{g}$ & Moments of inertia of the pinion and gear \\
$K_{z}$ & Variable mesh stiffness \\
$K_{y}$ & Stiffness of the supports of the gears \\
$K_{m}$ & Averaged mesh stiffness \\
$2 K_{a}$ & Variation rate of mesh stiffness \\
$\varphi$ & Phase displacement \\
$\varepsilon$ & Contact ratio factor \\
\hline
\end{tabular}

\section{Introduction}

In theory, many models are elaborated in order to interpret faithfully the real conditions for predicting the dynamic behavior of gear transmissions. Historically, models are based on mass-spring-damper model with concentrated parameters, considering the gear as two rigid

\footnotetext{
${ }^{a}$ Corresponding author: moustapha_merzoug@yahoo.fr
}

cylinders linked by constant or varying mesh stiffness. Many of these models are favouring the torsional effects and are neglecting those which are flexural in order to obtain a model with only one degree of freedom [1]. In reference [2], a numerical model have been developed allowing the defects effect simulation on gear teeth and two kinds of defects were analyzed: cracks and surface defects. By taking in consideration the damping, the relation between the damping effect and the applied charge has been highlighted [3]. In references [4,5], the authors have adopted a dynamic modeling of all elastic components of the transmission (gears, shafts, bearings and housing) based on finite elements discretization.

In the other hand, many researchers have developed techniques allowing the gear defects detection and diagnosis, based on the vibration signals analysis [6-14]. In the gear mechanical system, the defects presence changes the vibration signature. In reference [7], the author has proposed the envelope analysis of a demodulated amplitude signal filtered around a harmonic in order to detect the crack defect presence. In reference [8], the WignerVille distribution has been used to detect the presence of the crack and other defects. In reference [9], the authors have developed a transfer function to filter the vibrating signals produced by gear housing, in order to recover the torsional vibrations coming directly from the gearing. In reference [10], the authors have used the wavelet transform in order to detect the eccentricity defects and 
have optimized some multi-resolution parameters. In reference [11], the authors have proposed a comparative study between the time- frequency approach, by applying the Kullback divergence on a spectrogram, and the cyclostationarity analysis, by using the spectral correlation. In reference [12], the authors have suggested indicators based on synchronous cepstral for the diagnosis of complex gear transmission. In reference [13], it is suggested to use an angular re-sampling technique combined with the instantaneous speed variation and transmission errors techniques for the detection of gears defects.

The gear transmissions defects monitoring is more complicated when the operating parameters of systems are variable especially for the speed and for the load. This kind of operating regime is prevalent in industry, for example the tramway, plane motors, wind turbine etc. Under these non-stationary operations, the amplitude and frequency components are always changing because of speed variation (or load variation). This affects clearly the classical diagnosis tools, which are not suitable for nonstationary analysis, and therefore a potential fault can be masked. Recently, this problematic was addressed by many research's works. In reference [14], it is found that the classical spectral indicator (gear frequency) is linear with the operating conditions indicators. This linearity forms a slope which is used as parameter for monitoring the planetary gearbox used in bucket wheel excavators. In reference [15], the authors present the results of an algorithm of vibration statistical analysis, applied on a test bench which simulates the wind turbine machine functioning under variable speed and load conditions. For the bearing defect monitoring in rotating machines that work under non-stationary regime, [16] the author has suggested a new indicator, based on accelerometer and optical encoder signals that are acquired simultaneously. An algorithm to estimate the instantaneous speed from optical encoder signal is applied. Then, each sample of accelerometer signal is divided by its corresponding instantaneous speed and the RMS value is applied to the resulting signal.

This work is based on simulated signals of the gears operating at variable speed regime. The aim is the proposition of relevant indicators on the gears state in variable regime. Among the most indicators used in the industry, we have the RMS value and the spectral analysis. These two indicators are adopted in this work and developed in the context of the variable regime. The paper is organized as follows: in Section 2 the adopted model is described. In Section 3 the dynamic response is detailed. In Section 4 the proposed indicators are given before showing their application to the simulated signals in the Section 5. A conclusion is given at the end.

\section{Description of the adopted model}

The most of behavior dynamic models of the gear transmissions use discrete models of mass-spring-damper. The gears are assimilated to rigid cylinders connected by a mesh stiffness which represents the elastic connection between teeth. The first works considered that the mesh stiffness is constant. This consideration requires to be taken in account in the dynamic equations of various external excitations, like the errors of form and assembly [17]. Actually, it is admitted that both internal excitation sources to be considered are those produced by the mesh stiffness fluctuation and those generated by the shapes gaps on the teeth $[18,19]$.

\section{Transmission dynamic response}

In general, the static error of gear transmission has many origins. Many works have been undertaken to analyze respectively the contribution of each of these origins to the vibrato-acoustic behavior of gear transmissions and to determine their frequency characteristics. These origins may be the geometry defects, resulting from the gears manufacture and the transmission assembling, or may be the teeth elastic deformation.

\subsection{Excitation induced by eccentricities default}

The eccentricity defect is due to the fact that the pitch circles of the wheels do not have their center on the rotation axis of the corresponding wheel. This defect may be associated with the incorrect assembly of the wheel on the shaft and/or the wheel and shaft manufacture. The eccentricity of a wheel is essentially characterized by the presence, in the spectrum of the transmission error, of the spectral component at the rotating frequency of the eccentric wheel and sidebands around the meshing frequency and its harmonics [20].

The transmission error $\delta(t)$ is the global elastic deformation of teeth at the contact point of a toothed wheels pair. The following equation presents its formula:

$$
\delta(t)=\theta_{p} R_{p}-\theta_{g} R_{g}+y_{p}-y_{g}+e_{p} \sin \left(\Omega_{1} t\right)-e_{g} \sin \left(\Omega_{2} t\right)
$$

\subsection{Motion equations}

The model showed in the Figure 1 can be formed into four equations, each one corresponds to a degree of freedom. The index $p$ is attributed to the pinion while the index $\mathrm{g}$ is attributed to the gear. $y_{p}$ and $y_{g}$ coordinates represent the displacements of the pinion and the gear. Angles $\theta_{p}$ and $\theta_{g}$ represent their rotations.

$$
\begin{gathered}
M_{p} \ddot{y}_{p}+\left(K_{y}+K_{z}\right) y_{p}-K_{z} R_{p} \theta_{p}-K_{z} y_{g}+K_{z} R_{g} \theta_{g}=0 \\
I_{p} \ddot{\theta}_{p}-K_{z} R_{p} y_{p}+K_{z} R_{p}^{2} \theta_{p}+K_{z} R_{p} y_{g}-K_{z} R_{p} R_{g} \theta_{g}=0 \\
M_{g} y_{g}-K_{z} y_{p}+K_{z} R_{p} \theta_{p}+\left(K_{y}+K_{z}\right) y_{g}-K_{z} R_{g} \theta_{g}=0 \\
I_{g} \ddot{\theta}_{g}+K_{z} R_{g} y_{p}-K_{z} R_{p} R_{g} \theta_{p}-K_{z} R_{g} y_{g}+K_{z} R_{g}^{2} \theta_{g}=0
\end{gathered}
$$



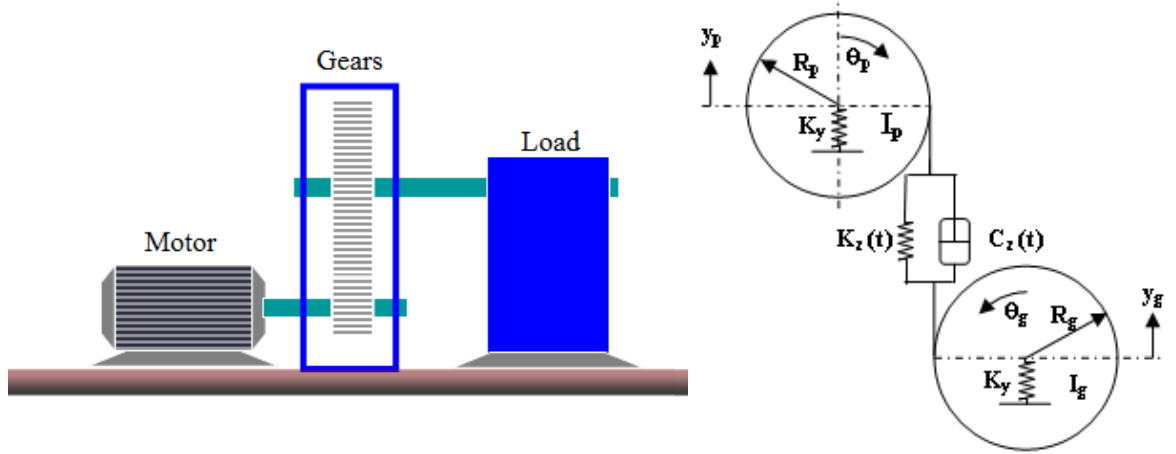

Fig. 1. Gear transmission dynamic model.

$M_{i}$ and $I_{i}$ with $i=p, g$, represent successively masses and moments of inertia of the pinion and gear. $K_{z}$ presents the variable mesh stiffness and $K_{y}$ the stiffness of the supports of the gears. Assuming that the mesh stiffness varies with the rotation of the drive shaft, its formula is given by [21]:

$$
\begin{aligned}
K_{z} & =K_{m}+2 K_{a} \sum_{i=1}^{\infty} a_{i} \sin \left(\Omega_{z} t\right)+b_{i} \cos \left(\Omega_{z} t\right) \\
a_{i} & =-\frac{2}{i \pi} \sin [i \pi(\varepsilon-2 \phi)] \sin (i \pi \varepsilon)
\end{aligned}
$$

and

$$
b_{i}=-\frac{2}{i \pi} \cos [i \pi(\varepsilon-2 \phi)] \sin (i \pi \varepsilon)
$$

\section{Suggested indicators}

RMS value is a very used indicator in the industry. It consists of measuring the level of static redressed energy of a vibration signal. Its estimation formula for a vibration signal is given by:

$$
R M S=\sqrt{\sum_{n=1}^{N} \frac{(x(n))^{2}}{N}}
$$

where $x(n)$ is the sample of vibration signal at time $n$ and $N$ is the number of samples.

The advantage of use the RMS value, in gears monitoring, is its implementation which is extremely easy. However, it is not very sensitive and in general we use it when the power of vibration signals in free fault mode are weak compared with the defect mode [22]. In a variable regime, this indicator is a linear function of rotational speed. The proposed method consists in eliminating the influence of speed rotation, which can mask a potential defect, by dividing the vibratory signal by the instantaneous frequency and then applying the RMS value. This new indicator was employed successfully for the diagnosis of bearings in variable speed regime [16] and will be the first indicator used in this work.

Spectral analysis is one of the most used methods in research and industry areas for gear transmissions diagnosis [22]. By analyzing the evolution of the amplitude of

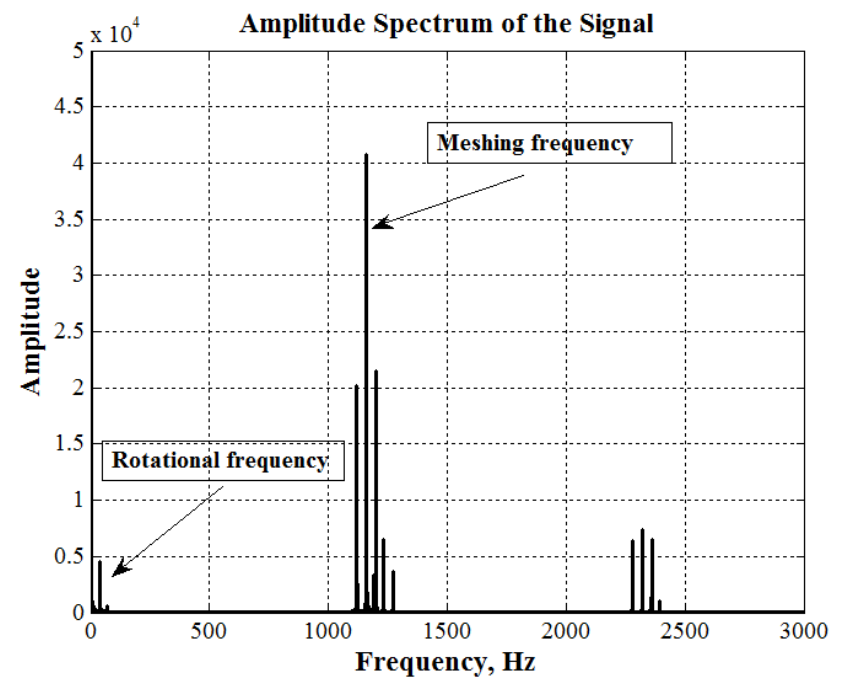

Fig. 2. Signal spectrum in the case of constant rotational speed.

rotation frequencies and gears frequencies, one can distinguish gear defects. However in the speed variable regime, the rotation speed variation deforms the spectrum components and therefore prevents the diagnosis. Speed variation causes a modulation of amplitude and frequency and therefore always changes the harmonics characteristics of gears. This amplitude and frequency modulation are considered as a linear function of speed.

The second proposed indicator in this article is the gear frequency normalized by the average speed. This consists in dividing the vibration signal in equal segments and for each one of them we detect meshing frequency amplitude (the big harmonic), then we divide this meshing frequency by the averaged speed estimated in this segment. Thus, the new indicator allows overcoming the speed variation impact on the classical spectral analysis.

\subsection{Application to the simulated signals}

Figure 2 represents the spectrum of the simulated signal of an eccentricity error on the pinion $\left(80 \times 10^{-6} \mathrm{~m}\right)$ in a constant regime where rotational frequency is $40 \mathrm{~Hz}$ and the teeth number is 29 . From this figure, one can note 


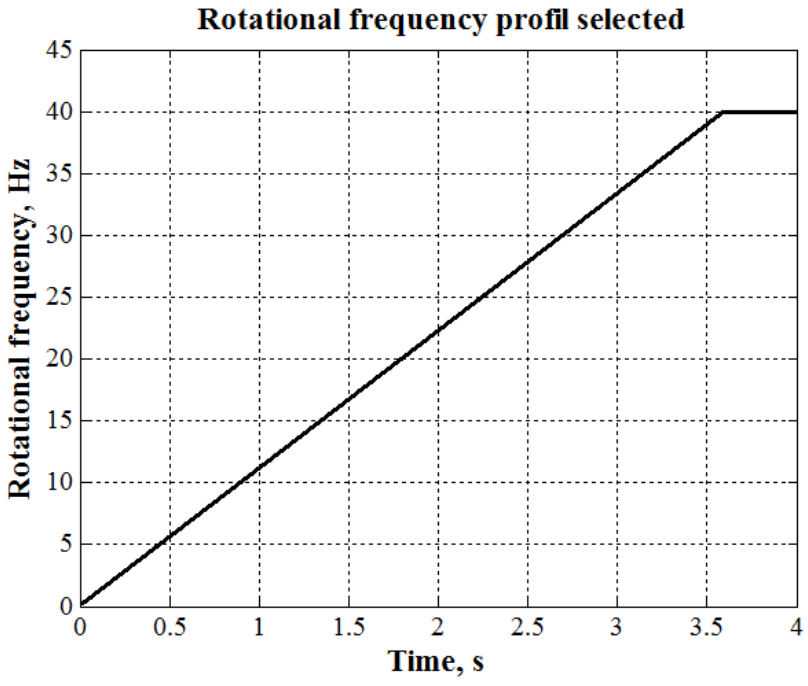

Fig. 3. The selected rotational frequency profile.

the meshing frequency predominance at $1160 \mathrm{~Hz}$ and its harmonics.

In order to test the proposed indicators, we have considered the simulation signals of gears in variable speed. The selected profile of the rotational frequency variation of the drive shaft is shown Figure 3. From this figure, the rotational frequency increases from 0 to $40 \mathrm{~Hz}$ in a first time, then it is constant for the remaining time. The choice of this profile is motivated by the existence in many industrial machines (wind turbine, navy propulsion systems, tramway) the starting phases and stops which are repeated infinitely.

For simulation signals, we define our rotational frequency. However, in general, we can use the following method: filtering pass-band around a characteristic frequency (meshing frequency, rotation frequency in the signal coming from optical encoder) then applying the Hilbert transform to get the analytical signal. From this analytic signal we estimate the phase, and by the phase derivation we get the instantaneous frequency. This algorithm can be applied to signals captured from optical encoders, installed in the majority of industrial machines, in order to estimate the instantaneous frequency. If we have a variable speed regime, we divide the signal into segments, where it is assumed that the speed is constant and then apply this algorithm.

Figure 4a presents the simulate signal of an eccentricity error on the pinion $\left(160 \times 10^{-6} \mathrm{~m}\right)$ and Figure $4 \mathrm{~b}$ presents its spectrum. From these figures; one can note that the temporal signal amplitude is modulated by the profile of the rotational frequency and the spectrum of the signal is difficult to interpret because of the speed variation that distorts the spectral components. Figure 5 presents the signal in time frequency domain; it is clear from this figure that the meshing frequency is linear with speed variation. Also its amplitude evolution is linear with speed variation.

To make a diagnosis with RMS value, we have divided the signal into segments of 1024 points and for each seg-

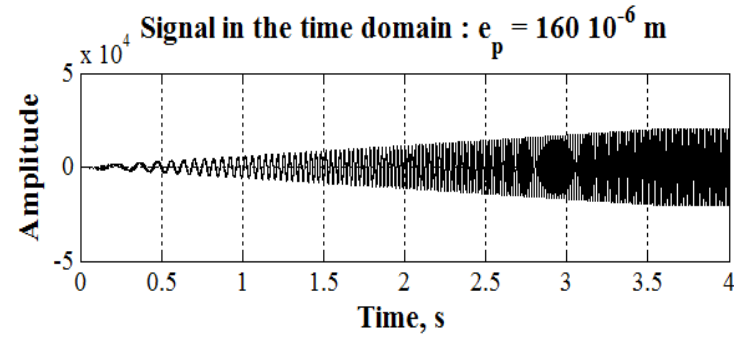

(a)

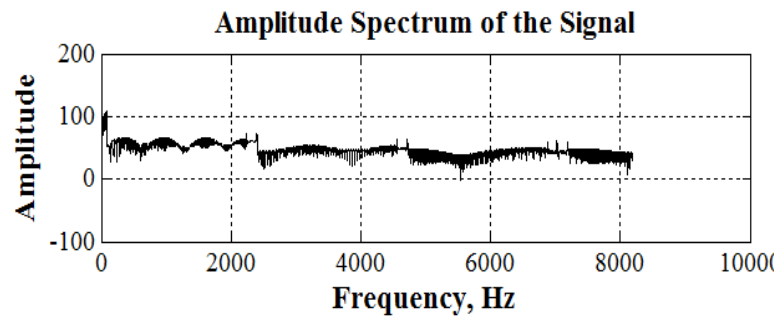

(b)

Fig. 4. (a) The simulated signal in time domain, (b) its spectrum for $e_{p}=160 \times 10^{-6} \mathrm{~m}$.

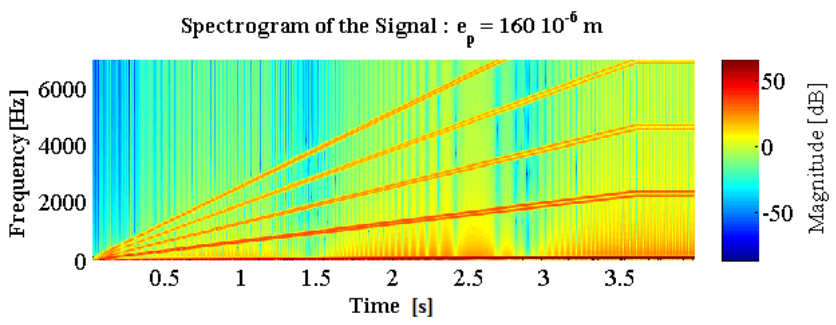

Fig. 5. The spectrogram of the simulated signal for $e_{p}=$ $160 \times 10^{-6} \mathrm{~m}$.

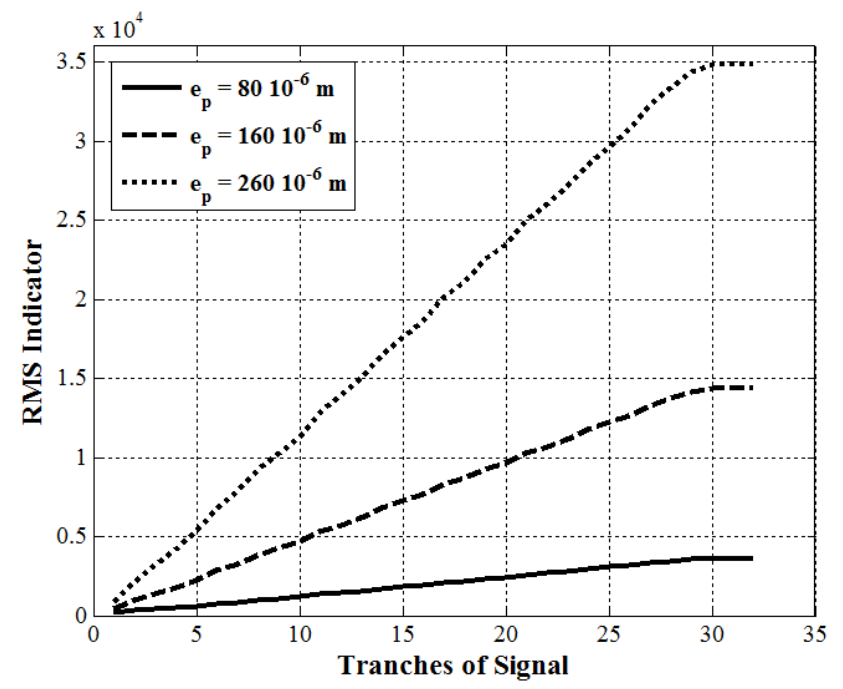

Fig. 6. RMS value.

ment the RMS value is calculated (Fig. 6). In this figure, we observe that the RMS value is a linear function of speed and therefore is strongly influenced by the speed variation, which prevents the diagnosis, for example, if a threshold is fixed at 2000, we may well see the three curves pass through this value. 


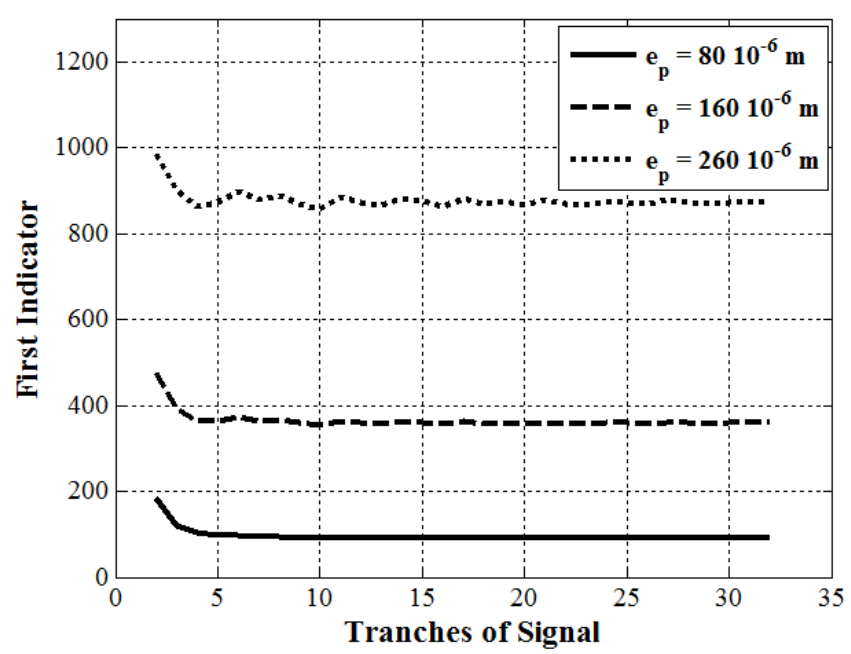

Fig. 7. First Indicator.

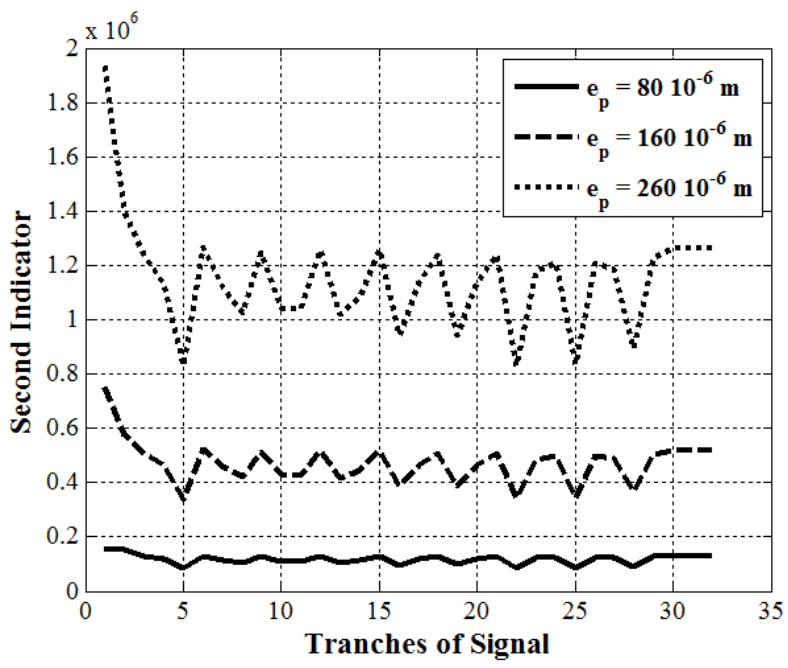

Fig. 8. Second Indicator.

Figures 7 and 8 present the two proposed indicators applied to the simulation signals. For the indicator 1, we can note that the three curves representing the different states of eccentricity error are practically constant and separable, whatever the rotational frequency variation (each block presents a different rotational speed). Likewise, the second indicator, extracted from the spectrogram, gives curves allowing to distinguish the three cases of eccentricity error. In order to highlight this result the thresholds can be fixed.

\section{Conclusion}

This work, based on numerical simulation, is a contribution in diagnosis of rotating machines in variable speed regime which the purpose is the relevant indicators search.

Numerical modeling has allowed to have a first approach of dynamic behavior of gear transmissions in variable speed regime. Indeed the simulated signals, in this paper, have essential characteristics of real signals, because that the chosen numerical model takes into account the gear transmission organs and can introduce different errors in order to study their influences in case where rotation frequency is variable. The proposed indicators are the RMS value applied to the vibration signal divided by its corresponding instantaneous frequency and the gear frequency, estimated in each segment, normalized by its corresponding averaging speed. Compared with the classical indicators we consider that the new indicators are promising, because the new indicators are able to diagnose a gear transmission in a variable speed regime and offer advantage to fix the thresholds.

\section{References}

[1] F. Chaari, T. Fakhfakh, M. Haddar, Simulation numérique du comportement dynamique d'une transmission par engrenages en présence de défauts de denture, Mechanics \& Industry 6 (2006) 625-633

[2] D. Palais, R. Guilbault, M. Thomas, A. Lakis, N. Mureithi, Numerical simulations of damaged gear vibrations Proceedings of the 27th seminar on machinery vibration. CMVA, Vancouver, 2009

[3] R. Guilbault, S. Lalonde, M. Thomas, Nonlinear damping calculation in cylindrical gear dynamic modelling, J. Sound Vib. 331 (2012) 2110-2128

[4] P. Ducret, Prédiction du bruit rayonné par les carters des transmissions à engrenages, Ph.D. Thesis, École centrale de Lyon, 1997

[5] E. Rigaud, J. Sabot, Effect of Elasticity of Shafts, Bearings, Casing and Couplings on the Critical Rotational Speeds of a Gearbox, Proceedings of the International Conference of Gears, Germany, 1996

[6] M. Badi, I. Esat, B. Paya, Neural network based fault detection using different signal processing techniques as pre-process, ASME Struct. Dyn. Vib. 70 (1995) 97-101

[7] P.D. McFadden, Advances in the vibration monitoring of gears and rolling element bearings, National symposium, 1985

[8] B.D. Forrester, Gear fault detection using the WignerVille distribution, The lnstitution of Engineers, Australia vibration and noise conference, 1990, pp. 296-299

[9] G. Dalpiaz, U. Meneghetti, Monitoring fatigue cracks in gear, NDT \& E International 24 (1991) 303-306

[10] M. Merzoug, A. Miloudi, X. Chiementin, Détection des défauts d'excentricités d'une transmission à engrenages par analyse en ondelettes, Congrès International sur les Risques Industriels, CIRI, 2009

[11] H. Laurent, M. Sidahmed, C. Doncar, Détection précoce d'endommagement de denture sur des trains d'engrenages droits par analyse vibratoire, Dix-septième colloque GRETSI, 1999

[12] D. Chikouche, A. Felkaoui, N. Haloui, Diagnostic précoce d'un réducteur à engrenage par analyse des signaux vibratoires à l'aide du cepstre synchrone, ICIEM, 2010

[13] S. Fedala, D. Remond, R. Zegadi, A. Felkaoui, Utilisation de l'erreur de transmission et de la vitesse angulaire instantanée pour le diagnostic vibratoire des 
défauts des engrenages, $3^{\text {ème }}$ Colloque Analyse Vibratoire Expérimentale, Blois, 20 et 21 Novembre 2012

[14] W. Bartelmus, F. Chaari, R. Zimroz, M. Haddar, Modelling of gearbox dynamics under time-varying nonstationary load for distributed fault detection and diagnosis, Eur. J. Mech. A/Solids 29 (2010) 637-646

[15] l. Villa, F. Renones, A. Peran, J. Miguel, Statistical fault diagnosis based on vibration analysis for gear test-bench under non-stationary conditions of speed and load, Mech. Syst. Signal Process. 29 (2012) 436-446

[16] K. Ait-Sghir, F. Bolaers, O. Cousinard, J.P. Dron, Spalling bearing defect in variable speed regime, Mechanics \& Industry 14 (2013) 129-136

[17] W.J. Pearce, M.A. Neilsen, W.S. Rouverol, Reducing Gear Noise Excitation, Proceedings of the 2nd World Congress on Gearing, Paris, 1986, 587-598
[18] A. Kassaï, J.P. Mathieu, G. Chahmirian, Sirènement de denture: Composantes non harmoniques de l'engrènement liées à la rectification par génération, Proceedings of the 3rd World Congress on Gearing and Power Transmissions, Paris, 1992

[19] D.B. Welbourn, Gear errors and their resultant noise spectra, Proceedings of Institution of Mechanical Engineers, 1969 184, Part 30, 131-139

[20] N. Driot, Étude de la dispersion vibroacoustique des transmissions par engrenages, Ph.D. thesis, École centrale de Lyon, 2002

[21] J. Lin, R.G. Parker, Mesh stiffness variation instabilities in two-stage gear system, J. Vib. Acoust. 124 (2002) 6876

[22] A. Boulenger, C. Pachaud, Diagnostic vibratoire en maintenance préventive, Dunod, Paris, 1998 